\title{
Les levures et les moisissures dans le fromage bleu de Cabrales
}

\author{
par \\ M. NUÑEZ, Margarita MEDINA, Pilar GAYA \\ et Carmen DIAS-AMADO*
}

\section{INTRODUCTION}

Dans une étude précédente sur l'évolution des principaux groupes microbiens [25], nous avons constaté au cours de l'affinage du fromage de Cabrales l'importance numérique de sa flore fongique.

Les levures peuvent participer à la maturation des fromages par la fermentation du lactose, la consommation de l'acide lactique, la production de substances stimulantes et par leurs activités protéolytique, lipolytique et de production de composés d'arôme.

Hartley et Jezeski [18] et Kanauchi et al. [19] ont étudié les levures du fromage Bleu et la flore levure du fromage de Roquefort est bien connue par les travaux de Devoyod et Sponem [11] et Galzin et al. [15]. Ces micro-organismes ont été étudiés aussi dans d'autres types de fromage : Camembert [20,35], Cantal [23], Chèvre [9], Fiore Sardo [14], Gorgonzola [28], Gruyère et Beaufort [1], Pecorino [31], Robiola [4, 5], Saint-Nectaire [7, 36], Saint-Paulin [12], Taleggio [37] et Telemes [16].

Les moisissures peuvent avoir également un rôle important dans l'affinage des fromages : elles contribuent à la neutralisation de la pâte, synthétisent des protéases et des lipases exocellulaires et forment des méthylcétones par la $\beta$-oxydation des acides gras. Des travaux ont été consacrés aux moisissures présentes dans divers types de fromage : Edam et Gouda [24], Provolone [29], Robiola [4, 5], Saint-Nectaire [8, 10], Taleggio [5] et Tome de Savoie [10].

Sala et al. [34] ont étudié les moisissures et Sala et Burgos [33] les levures dans le fromage de Cabrales affiné et ont trouvé des sou-

* Département de Biochimie et de Microbiologie. C.R.I.D.A. 06. I.N.I.A. Carretera de la Coruna $\mathrm{Km} 7$ - Apdo. 8111 - Madrid (Espagne). 
ches appartenant aux espèces Penicillium roqueforti, $P$. chrysogenum, Mucor mucedo, Kluyveromyces lactis, Candida krusei et Trichosporon penicillatum. Deux travaux $[26,27]$ ont été publiés récemment sur les principales espèces bactériennes présentes au cours de l'affinage dans le fromage de Cabrales, mais aucune étude n'a encore été faite sur l'évolution du nombre et des espèces de levures et de moisissures pendant la maturation de ce type de fromage.

En raison de l'importance numérique de la flore fongique rencontrée au cours de l'affinage du fromage de Cabrales, nous avons été amenés à préciser l'identité des levures et des moisissures afin de mieux comprendre sa contribution aux phénomènes biochimiques complexes de la maturation de ce fromage bleu.

\section{MATERIEL ET METHODES}

\section{Dénombrements et isolements des souches}

Nous avons suivi l'évolution de la flore fongique au cours de la maturation de deux fabrications traditionnelles (A et B) de fromage bleu de Cabrales. Les échantillons de lait ont été pris avant l'emprésurage, les échantillons de caillé à la mise en moules et les échantillons de la surface et de l'intérieur du fromage à divers stades de l'affinage (jusqu'à 4 mois).

Des dilutions décimales ont été ensemencées $(0,1 \mathrm{ml})$ sur Potato Dextrose Agar (Difco) acidifié à $\mathrm{pH}$ 3,5 par l'acide tartrique à 10 p. 100 [3]. Les dénombrements des colonies ont été réalisés après $96 \mathrm{~h}$ d'incubation à $26^{\circ} \mathrm{C}$.

Six colonies ont été prélevées au hasard sur les boîtes de Pétri d'une dilution appropriée. Après purification, les souches ont été conservées sur gélose YM inclinée de Wickerham [39] à $4^{\circ} \mathrm{C}$.

\section{Identification}

\section{a) LEVURES}

Les souches de levures isolées ont été identifiées selon la classification et les techniques proposées par Lodder (21).

1. Morphologie et caractères culturaux

Un examen microscopique des cultures sur bouillon YM de Wickerham [39] après $72 \mathrm{~h}$ d'incubation à $24^{\circ} \mathrm{C}$ a été utilisé pour l'observation de la morphologie des levures. Les caractères culturaux ont été notés sur milieu YM en boîte de Petri et en gélose inclinée, après 3 jours à $24^{\circ} \mathrm{C}$. 


\section{Formation de filaments mycéliens}

Une technique de culture sur milieu gélosé YM, entre lame et lamelle stériles, a été employée. Les cultures ont été examinées après $6 \mathrm{j}$ d'incubation à $24^{\circ} \mathrm{C}$.

\section{Formation d'ascospores}

La formation d'ascospores a été décelée sur la gélose acétate de Mc Clary et al. [22] incubée à $24^{\circ} \mathrm{C}$ et observée chaque semaine pendant 28 j selon la technique de Wickerham [39].

\section{Croissance en absence de vitamines}

Le milieu Bacto-Vitamin Free Yeast Base (Difco) a été employé, selon la méthode de Wickerham [39]. La lecture était faite après $7 \mathrm{j}$ d'incubation à $24^{\circ} \mathrm{C}$.

\section{Assimilation des composés organiques}

La technique de Wickerham et Burton [40.] sur milieu liquide (Bacto-Yeast Nitrogen Base, Difco) a été suivie. Les composés à tester étaient stérilisés par filtration. La lecture des résultats eut lieu tous les $7 \mathrm{j}$, pendant 3 semaines.

\section{Fermentation des sucres}

La fermentation des sucres était décelée sous cloche de Durham en milieu liquide. Les cultures étaient incubées à $24^{\circ} \mathrm{C}$ et observées les $3^{\mathrm{e}}, 7^{\mathrm{e}}, 14^{\mathrm{e}}, 21^{\mathrm{e}}$ et $28^{\mathrm{e}} \mathrm{j}$.

\section{Assimilation des nitrates}

La méthode décrite par Wickerham [38] a été utilisée. La lecture était faite après $7 \mathrm{j}$ à $24^{\circ} \mathrm{C}$. Si le résultat était positif, un deuxième tube était ensemencé pour confirmation.

\section{Croissance en présence de différentes concentrations de $\mathrm{NaCl}$}

Le milieu de base utilisé était le bouillon YM additionné de $\mathrm{NaCl}$ à différentes concentrations : $5-20 \mathrm{p} .100(\mathrm{p} / \mathrm{v})$. La turbidité des cultures était examinée après $7 \mathrm{j}$ d'incubation à $24^{\circ} \mathrm{C}$, à l'aide d'un appareil Spectronic 20 (Bausch et Lomb).

\section{b) MOISISSURes}

Les souches de moisissures isolées ont été identifiées d'après Ainsworth et al. [2]. Pour les souches appartenant au genre Penicillium, nous avons suivi la classification de Raper et Thom [30] et les critères de Samson et al. [32].

\section{Morphologie et caractères culturaux}

La morphologie des moisissures a été observée par l'examen microscopique des cultures sur lame sur gélose YM les $4^{\mathrm{e}}$ et $6^{\mathrm{e}} \mathrm{j}$ d'incubation à $24^{\circ} \mathrm{C}$. Les caractères culturaux ont été notés sur les milieux 


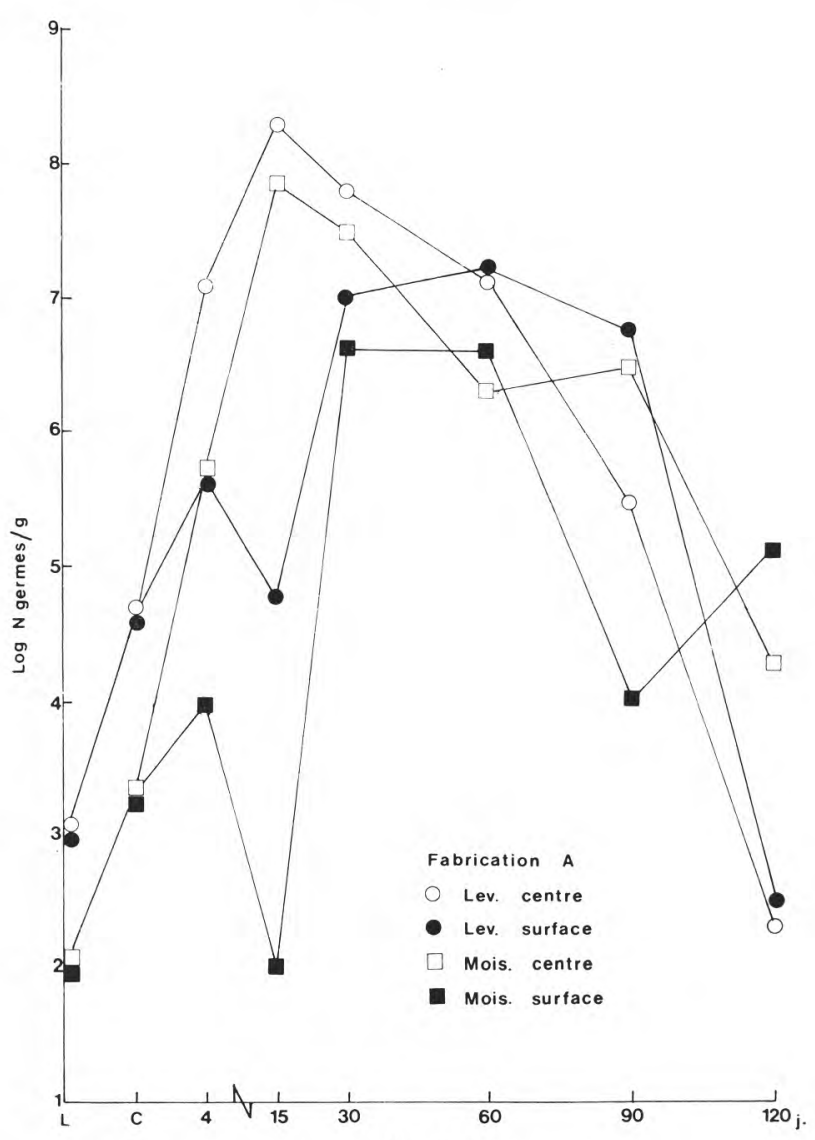

fig. 1

Evolution du nombre des levures et des moisissures au cours de l'affinage du fromage de Cabrales. Fabrication A.

YM et Czapek-Dox en boîte de Pétri et en gélose inclinée, les $6^{\mathrm{e}}$ et $14^{\mathrm{e}} \mathrm{j}$ d'incubation à $24^{\circ} \mathrm{C}$.

2. Croissance en présence de différentes concentrations d'acide acétique, d'acide lactique et de $\mathrm{NaCl}$

La mise en culture des moisissures en présence de 0,5 p. 100 (p/v) d'acide acétique et de 4 p. $100(\mathrm{p} / \mathrm{v})$ d'acide lactique préconisée par Engel et Teuber [13] pour la confirmation de Penicillium roqueforti a été réalisée. Des concentrations supérieures (1 p.100 d'acide acétique et 6 p. 100 d'acide lactique) ont été aussi employées.

Pour la croissance en présence de différentes concentrations de $\mathrm{NaCl}$ on a suivi la méthode décrite pour les levures. 


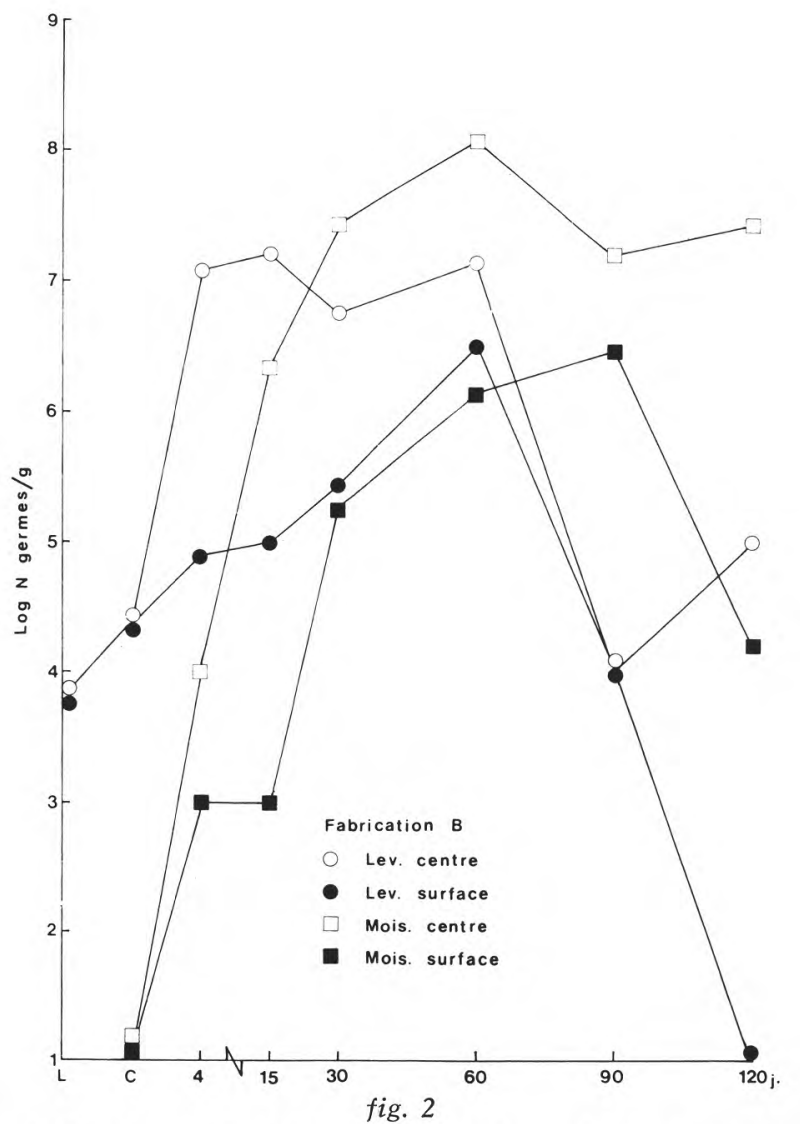

Evolution du nombre des levures et des moisissures au cours de l'affinage du fromage de Cabrales. Fabrication B.

\section{Assimilation du lactose et de l'acide lactique}

L'assimilation du lactose et de l'acide lactique par les moisissures a été étudiée sur bouillon Czapek-Dox, dans lequel le saccharose a été remplacé par 2 p. 100 de lactose ou par 2 p. 100 (p/v) d'acide lactique. Les lectures étaient faites après 7 et $14 \mathrm{j}$ à $24^{\circ} \mathrm{C}$.

\section{RESULTATS}

\section{Evolution du nombre des levures et des moisissures}

L'évolution de la flore fongique au cours de la maturation de deux fabrications (A et B) de fromage bleu de Cabrales a été indiquée dans les figures 1 et 2 . 
Le nombre des levures au centre du fromage augmente pendant la fabrication, le salage ( $3^{\mathrm{e}}$ et $\left.4^{\mathrm{e}} \mathrm{j}\right)$ et le premier affinage $\left(5^{\mathrm{e}}-15^{\mathrm{e}} \mathrm{j}\right)$ et atteint son maximum vers le $15^{\mathrm{e}} \mathrm{j}: 1,9 \times 10^{8} / \mathrm{g}$ dans le fromage $\mathrm{A}$ et $1,6 \times 10^{7} / \mathrm{g}$ dans le fromage B. A ce moment-là, les levures sont, après les streptocoques lactiques, le groupe microbien le plus important. Au cours du deuxième affinage, leur nombre diminue lentement jusqu'au $60^{\mathrm{e}}$ jour et plus rapidement durant les 2 derniers mois.

A la surface du fromage, le niveau le plus élevé est atteint vers le $60^{\mathrm{e}}$ jour : $1,7 \times 10^{7}$ levures/g dans le fromage $\mathrm{A}$ et $3,2 \times 10^{6} / \mathrm{g}$ dans le fromage $B$.

Les moisissures arrivent à leur maximum au centre du fromage le $15^{\mathrm{e}}$ jour dans la fabrication $\mathrm{A}\left(7,3 \times 10^{7} / \mathrm{g}\right)$ et le $60^{\mathrm{e}}$ jour dans la fabrication B $\left(1,2 \times 10^{8} / \mathrm{g}\right)$.

A l'extérieur du fromage, le développement des moisissures continue pendant presque tout le deuxième affinage : dans la fabrication $\mathrm{A}$, le maximum est atteint le $30^{\mathrm{e}} \mathrm{j}$ et dans la fabrication $\mathrm{B}$, le $90^{\mathrm{e}} \mathrm{j}$.

\section{Identification des levures}

Lors de notre étude, 103 souches de levures ont été isolées pendant la fabrication et l'affinage de deux cuviers de fromage de Cabrales. La répartition des différentes espèces de levures, d'après leur origine, est donnée dans le tableau 1.

\section{TABLEAU 1}

Répartition des levures isolées du fromage de Cabrales

\begin{tabular}{|c|c|c|c|c|c|c|}
\hline \multirow{2}{*}{ Espèce } & \multirow{2}{*}{$\begin{array}{c}\text { Lait } \\
+ \\
\text { caillé }\end{array}$} & \multicolumn{2}{|c|}{ Premier affinage } & \multicolumn{2}{|c|}{ Deuxième affinage } & \multirow{2}{*}{ Total } \\
\hline & & I & $\mathrm{E}$ & I & $\mathrm{E}$ & \\
\hline Pichia fermentans & 7 & 3 & 2 & 4 & - & 16 \\
\hline P. kluyveri & - & 2 & 2 & 2 & - & 6 \\
\hline P. membranaefaciens & - & 12 & 2 & 8 & - & 22 \\
\hline Torulopsis candida & - & - & 7 & - & 9 & 16 \\
\hline Debaryomyces hansenii & - & - & 1 & 1 & 13 & 15 \\
\hline Trichosporon capitatum & 12 & - & 3 & - & - & 15 \\
\hline Saccharomyces chevalieri & - & - & - & 1 & - & 1 \\
\hline Sacch. rosei & - & - & 1 & - & - & 1 \\
\hline Sacch. rouxii & - & 1 & - & - & - & 1 \\
\hline Sacch. unisporus & 2 & - & 1 & - & - & 3 \\
\hline Candida ingens & - & 2 & - & 1 & - & 3 \\
\hline C. rugosa & - & 1 & - & - & - & 1 \\
\hline Kluyveromyces lactis & - & 1 & 2 & - & - & 3 \\
\hline
\end{tabular}

I : intérieur du fromage ; $\mathrm{E}$ : extérieur du fromage. 


\section{TABLEAU 2}

Assimilation et fermentation des différents composés par les principales espèces de levures isolées du fromage de Cabrales

\begin{tabular}{|c|c|c|c|c|c|c|}
\hline Espèce & 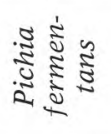 & 离竎 & 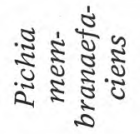 & 竞: & 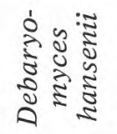 & 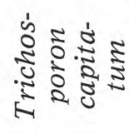 \\
\hline Nombre de souches & 16 & 6 & 22 & 16 & 15 & 15 \\
\hline \multicolumn{7}{|l|}{ Assimilation: } \\
\hline Glucose & + & + & + & + & + & + \\
\hline Galactose & - & - & - & + & + & + \\
\hline Sorbose & - & - & - & + & + & + \\
\hline Maltose & - & - & 1 & + & + & 1 \\
\hline Saccharose & - & - & - & + & + & - \\
\hline Cellobiose & - & - & - & + & + & 1 \\
\hline Tréhalose & - & - & - & + & + & - \\
\hline Lactose & - & - & - & 14 & 12 & - \\
\hline Mélibiose & - & - & - & 7 & 9 & - \\
\hline Raffinose & - & - & - & + & 14 & - \\
\hline Mélézitose & - & - & - & + & 14 & - \\
\hline Amidon & - & - & - & 6 & 9 & - \\
\hline Inuline & - & - & - & 12 & 1 & - \\
\hline Xylose & + & - & 15 & + & + & - \\
\hline L-arabinose & - & - & - & + & + & - \\
\hline D-arabinose & - & - & - & - & - & - \\
\hline Ribose & - & - & - & - & 1 & + \\
\hline Rhamnose & - & - & - & 15 & 13 & - \\
\hline Glucosamine & 15 & - & 8 & - & - & - \\
\hline Erythritol & - & - & - & 10 & 6 & + \\
\hline Galactitol & - & - & - & - & - & - \\
\hline Mannitol & - & - & - & + & 14 & + \\
\hline Glucitol & - & - & - & + & + & + \\
\hline$\alpha$-M-D-glucoside & - & - & - & + & 14 & - \\
\hline Salicine & - & - & - & + & 14 & - \\
\hline Ac. pyruvique & + & + & 21 & - & 3 & + \\
\hline Ac. lactique & + & + & + & + & + & + \\
\hline Inositol & - & - & - & - & - & - \\
\hline Nitrates & - & - & - & - & - & - \\
\hline \multicolumn{7}{|l|}{ Fermentation: } \\
\hline Glucose & + & + & 15 & 5 & 1 & - \\
\hline Galactose & - & - & - & - & - & - \\
\hline Saccharose & - & - & - & - & - & - \\
\hline Maltose & - & - & - & - & - & - \\
\hline Lactose & - & - & - & - & - & - \\
\hline Raffinose & - & - & - & - & - & - \\
\hline
\end{tabular}

Les chiffres indiquent le nombre de souches qui donnent une réaction + . 
Les souches isolées appartiennent aux genres Pichia, Torulopsis, Debaryomyces, Trichosporon, Saccharomyces, Candida et Kluyveromyces.

L'assimilation et la fermentation des différents composés par les principales espèces de levures isolées du Cabrales sont indiquées dans le tableau 2. Aucune des souches étudiées ne se développe dans le milieu Bacto-Vitamin Free Yeast Base.

Genre Pichia Hansen : 44 souches de levures ont été identifiées comme appartenant à ce genre. Par leurs caractères morphologiques, culturaux et biochimiques, 16 souches ont été considérées comme $P$. fermentans Lodder, 6 souches comme P. kluyveri Bedford et 22 souches comme $P$. membranaefaciens Hansen. Une souche de $P$. membranaefaciens assimilait faiblement le maltose.

Genre Torulopsis Berlese : 16 souches du genre Torulopsis ont été isolées et identifiées comme $T$. candida (Saito) Lodder, puisqu'elles coïncident avec la description de l'espèce. Cinq souches fermentaient très faiblement le glucose.

Genre Debaryomyces Lodder et Kreger-van Rij nom. conserv. : les 15 souches incluses dans ce genre ont été considérées comme appartenant à $D$. hansenii (Zopf) Lodder et Kreger-van Rij. Quelques souches ne correspondent pas exactement avec tous les caractères biochimiques indiqués par Lodder [21], mais elles ont la morphologie et les caractères culturaux de l'espèce. L'amidon est assimilé seulement par 9 souches et le glucose fermenté faiblement par 1. Une souche atypique n'assimilait pas le raffinose, le mélézitose, le $\alpha$-méthylD-glucoside ni la salicine.

Genre Trichosporon Berend : 15 isolements de levures appartenaient à ce genre, mais elles ne correspondaient avec aucune des espèces indiquées par Lodder. Elles ont été incluses dans l'espèce $T r$ capitatum Diddens et Lodder, bien que l'assimilation de ribose, érythritol et mannitol était positive.

Genre Saccharomyces Meyen emend. Reess : 6 souches de levures ont été incluses dans ce genre. Une souche capable d'assimiler le glucose, le galactose, le saccharose, le tréhalose, le raffinose, l'inuline, le xylose, le mannitol, le glucitol et l'acide lactique et de fermenter le glucose, le galactose et le saccharose a été considérée comme Sacch. chevalieri Guilliermond, quoiqu'elle ne fermentait pas le raffinose. Une souche qui assimilait le glucose, le galactose, le saccharose, le raffinose, l'inuline, le xylose, le mannitol, le sorbitol et l'acide lactique, qui fermentait le glucose et qui se développait en présence de 50 p. 100 de glucose a été incluse dans l'espèce Sacch. rosei (Guilliermond) Lodder et Kreger-van Rij. Une autre souche capable d'assimiler le glucose, le galactose, le sorbose, le maltose, le xylose, le mannitol, le sorbitol, l'acide pyruvique et l'acide lactique et de se développer en présence de 50 p. 100 de glucose a été considérée comme Sacch. rouxii Boutroux. Les 3 souches restantes qui assimilaient seule- 
ment le glucose et le galactose ont été incluses dans l'espèce Sacch. unisporus Jörgensen.

Genre Candida Berkhout : 4 des souches de levures isolées appartenaient à ce genre. Trois souches capables d'assimiler le glucose, le galactose, le sorbose (2 souches), le mannitol, le sorbitol, l'acide pyruvique et l'acide lactique ont été considérées comme $C$. ingens van der Walt et van Kerken. Une souche qui assimilait le glucose, le galactose, le sorbose, le xylose, le L-arabinose, le mannitol, le sorbitol, l'acide pyruvique et l'acide lactique a été incluse dans l'espèce C. rugosa (Anderson) Diddens et Lodder.

Genre Kluyveromyces van der Walt emend, van der Walt : les 3 souches de levures incluses dans ce genre assimilaient le glucose, le galactose, le sorbose, le maltose, le saccharose, le cellobiose, le tréhalose, le lactose, le raffinose, le mélézitose, le mannitol, le glucitol, le $\alpha$-méthyl-D-glucoside (2 souches), la salicine (2 souches), l'acide pyruvique et l'acide lactique et fermentaient le glucose, le galactose (1 souche), le saccharose (1 souche) et le lactose. Elles ont été considérées comme $K$. lactis (Dombrowski) van der Walt.

\section{Croissance des levures à différentes concentrations de $\mathrm{NaCl}$}

Le développement des levures isolées du fromage de Cabrales en présence de $5-20$ p. 100 de $\mathrm{NaCl}$ en bouillon YM a été étudié. Dans le tableau 3 nous avons indiqué les concentrations maximales de $\mathrm{NaCl}$ auxquelles les différentes espèces de levures étaient capables de se développer, d'après leur origine.

Aucune des souches étudiées ne se développait avec 17,5 p. 100 de $\mathrm{NaCl}$. Neuf souches de $D$. hansenii et 7 souches de $T$. candida, les 16 isolées de l'extérieur du fromage, poussaient avec 15 p. $100 \mathrm{de} \mathrm{NaCl}$. En présence de 12,5 p. 100 de $\mathrm{NaCl}, 14$ souches de $D$. hansenii, 11 souches de $T$. candida, 6 souches de $T r$. capitatum, 3 souches de $K$. lactis, 1 souche de Sacch. chevalieri et 1 souche de Sacch. rosei se développaient.

\section{Identification des moisissures}

Soixante-deux souches de moisissures isolées à partir du lait, du caillé et du fromage à divers stades de l'affinage ont été identifiées comme appartenant aux genres Penicillium, Geotrichum et Paecilomyces. La répartition des souches d'après leur origine est montrée dans le tableau 4. Leur croissance dans le bouillon Czapek-Dox additionné de l'acide acétique ou de l'acide lactique est indiquée dans le tableau 5.

Genre Penicillium Link : 50 souches de moisissures appartenaient au genre Penicillium. L'espèce $P$. roqueforti Thom est, avec 34 souches, la plus fréquemment isolée. Ces 34 souches se développaient en présence de 0,5 et de 1 p. 100 d'acide acétique. Douze souches 


\section{TABLEAU 3}

Tolérance des levures isolées du fromage de Cabrales au $\mathrm{NaCl}$

\begin{tabular}{|c|c|c|c|c|c|}
\hline \multirow{2}{*}{ Espèce } & \multirow{2}{*}{$\begin{array}{c}\text { Lait } \\
+ \\
\text { caillé }\end{array}$} & \multicolumn{2}{|c|}{ Premier affinage } & \multicolumn{2}{|c|}{ Deuxième affinage } \\
\hline & & I & $\mathrm{E}$ & I & $\mathrm{E}$ \\
\hline $\begin{array}{l}\text { P. fermentans } \\
\text { P. kluyveri } \\
\text { P. membranaefaciens } \\
\text { T. candida } \\
\text { D. hansenii } \\
\text { Tr. capitatum } \\
\text { Sacch. chevalieri } \\
\text { Sacch. rosei } \\
\text { Sacch. rouxii } \\
\text { Sacch. unisporus } \\
\text { C. ingens } \\
\text { C. rugosa } \\
\text { K. lactis }\end{array}$ & $\begin{array}{c}7,9 \pm 0,9 \\
\overline{-} \\
\overline{-} \\
10,8 \pm 1,2 \\
\overline{-} \\
\overline{-} \\
8,8 \pm 1,8 \\
= \\
=\end{array}$ & $\begin{array}{c}7,5 \pm 0,0 \\
8,8 \pm 1,8 \\
7,9 \pm 1,0 \\
- \\
- \\
- \\
- \\
7,5 \pm 0,0 \\
- \\
7,5 \pm 0,0 \\
7,5 \pm 0,0 \\
12,5 \pm 0,0\end{array}$ & $\begin{array}{r}7,5 \pm 0,0 \\
10,0 \pm 0,0 \\
7,5 \pm 0,0 \\
11,4 \pm 2,0 \\
12,5 \pm 0,0 \\
11,7 \pm 1,4 \\
- \\
12,5 \pm 0,0 \\
10,0 \pm 0,0 \\
- \\
- \\
12,5 \pm 0,0\end{array}$ & $\begin{array}{r}7,5 \pm 0,0 \\
7,5 \pm 0,0 \\
8,1 \pm 1,2 \\
- \\
7,5 \pm 0,0 \\
=- \\
12,5 \pm 0,0 \\
- \\
- \\
7,5 \pm 0,0 \\
-\end{array}$ & $\begin{array}{c}\overline{-} \\
\overline{-} \\
13,9 \pm 1,8 \\
14,2 \pm 1,2 \\
= \\
= \\
= \\
= \\
= \\
=\end{array}$ \\
\hline
\end{tabular}

I : intérieur du fromage ; E : extérieur du fromage.

Les chiffres indiquent la concentration (px100/v) maximale de $\mathrm{NaCl}$ (moyenne et déviation standard) à laquelle chaque espèce est capable de se développer, d'après son origine.

\section{TABLEAU 4}

Répartition des moisissures isolées du fromage de Cabrales

\begin{tabular}{|c|c|c|c|c|c|c|}
\hline \multirow{2}{*}{ Espèce } & \multirow{2}{*}{$\begin{array}{c}\text { Lait } \\
+ \\
\text { caillé }\end{array}$} & \multicolumn{2}{|c|}{ Premier affinage } & \multicolumn{2}{|c|}{ Deuxième affinage } & \multirow{2}{*}{ Total } \\
\hline & & I & $\mathrm{E}$ & I & $\mathrm{E}$ & \\
\hline Penicillium frequentans & - & - & - & 2 & 10 & 12 \\
\hline$P$. purpurogenum & - & - & - & 1 & - & 1 \\
\hline P. roqueforti & - & 1 & - & 21 & 12 & 34 \\
\hline$P$. terrestre & - & 1 & - & - & 1 & 2 \\
\hline Penicillium sp. & - & - & - & - & 1 & 1 \\
\hline Geotrichum candidum & 3 & 3 & - & 5 & - & 11 \\
\hline Paecilomyces varioti & - & - & - & - & 1 & 1 \\
\hline
\end{tabular}

I : intérieur du fromage ; E : extérieur du fromage. 
TABLEAU 5

Croissance des moisissures à différentes concentrations d'acide acétique et d'acide lactique

\begin{tabular}{|c|c|c|c|c|c|}
\hline \multirow{2}{*}{ Espèce } & \multirow{2}{*}{$\begin{array}{c}\text { Nombre } \\
\text { de } \\
\text { souches }\end{array}$} & \multicolumn{2}{|c|}{ Acide acétique } & \multicolumn{2}{|c|}{ Acide lactique } \\
\hline & & 0,5 p. 100 & 1 p. 100 & 4 p. 100 & 6 p. 100 \\
\hline Penicillium frequentans & 12 & - & - & - & - \\
\hline P. purpurogenum & 1 & - & - & - & - \\
\hline P. roquefort $i$ & 34 & + & + & + & - \\
\hline P. terrestre & 2 & - & - & - & - \\
\hline Penicillium sp. & 1 & - & - & - & - \\
\hline Geotrichum candidum & 11 & - & - & - & - \\
\hline Paecilomyces varioti & 1 & - & - & + & - \\
\hline
\end{tabular}

identifiées comme $P$. frequentans Westling, 2 souches de $P$. terrestre Jensen, 1 souche de $P$. purpurogenum Fleroff-Stoll et 1 souche de Penicillium sp. ne poussaient pas avec 0,5 p. 100 d'acide acétique ni avec 4 p. 100 d'acide lactique. Toutes les souches du genre Penicillium assimilaient le lactose et l'acide lactique après $7 \mathrm{j}$, à l'exception d'une souche de $P$. terrestre qui n'assimilait l'acide lactique qu'après $14 \mathrm{j}$.

Genre Geotrichum Link : les 11 souches incluses dans ce genre ont été identifiées comme G. candidum Link. Elles ne poussaient pas avec 0,5 p. 100 d'acide acétique ni avec 4 p. 100 d'acide lactique. Quatre souches assimilaient le lactose après $7 \mathrm{j}$ et les 11 souches le lactose et l'acide lactique après $14 \mathrm{j}$.

Genre Paecilomyces Bainier : une souche a été isolée et identifiée comme $P$. varioti. Cette souche se développait en présence de 4 p. 100 d'acide lactique, mais pas avec 0,5 p. 100 d'acide acétique. Elle assimilait le lactose et l'acide lactique après $7 \mathrm{j}$ d'incubation.

\section{Croissance des moisissures à différentes concentrations de $\mathrm{NaCl}$}

Les résultats de la croissance des moisissures en présence de différentes concentrations de $\mathrm{NaCl}$ sont indiqués dans le tableau 6 . Toutes les souches de P. frequentans se développaient avec 20 p. 100 de $\mathrm{NaCl}$ après 7 jours et les souches de $P$. terrestre et Penicillium sp. après $14 \mathrm{j}$. Seulement une souche de $P$. roqueforti poussait en présence de 15 p. 100 de $\mathrm{NaCl}$, mais les 34 souches se développaient avec 12,5 p. 100 . 


\section{TABLEAU 6}

Tolérance des moisissures isolées du fromage de Cabrales au $\mathrm{NaCl}$

\begin{tabular}{|c|c|c|c|c|c|}
\hline \multirow{2}{*}{ Espèce } & \multirow{2}{*}{$\begin{array}{c}\text { Lait } \\
+ \\
\text { caillé }\end{array}$} & \multicolumn{2}{|c|}{ Premier affinage } & \multicolumn{2}{|c|}{ Deuxième affinage } \\
\hline & & I & $\mathrm{E}$ & I & $\mathrm{E}$ \\
\hline $\begin{array}{l}\text { P. frequentans } \\
P \text {. purpurogenum } \\
\text { P. roqueforti } \\
\text { P. terrestre } \\
\text { Penicillium } \mathrm{sp} . \\
\text { G. candidum } \\
\text { Paec. varioti }\end{array}$ & $\begin{array}{c}\overline{-} \\
\overline{-} \\
\bar{z} \\
7,5 \pm 0,0\end{array}$ & $\begin{array}{c}\overline{-} \\
12,5 \pm 0,0 \\
20,0 \pm 0,0 \\
\overline{-} \\
7,5 \pm 0,0 \\
-\end{array}$ & $\begin{array}{l}- \\
\overline{-} \\
\overline{-} \\
\overline{-}\end{array}$ & $\begin{array}{r}20,0 \pm 0,0 \\
7,5 \pm 0,0 \\
12,5 \pm 0,0 \\
- \\
- \\
7,5 \pm 0,0 \\
-\end{array}$ & $\begin{array}{r}20,0 \pm 0,0 \\
- \\
12,7 \pm 0,7 \\
20,0 \pm 0,0 \\
20,0 \pm 0,0 \\
- \\
7,5 \pm 0,0\end{array}$ \\
\hline
\end{tabular}

I : intérieur du fromage ; E : extérieur du fromage.

Les chiffres indiquent la concentration (px100/v) maximale de $\mathrm{NaCl}$ (moyenne et déviation standard) à laquelle chaque espèce est capable de se développer, d'après son origine.

\section{DISCUSSION}

\section{a) LES LEVURES}

\section{La flore levure du lait et du caillé}

La composition de la flore levure du lait et du caillé dans la fabrication du fromage de Cabrales est très variable selon les cuviers. Ainsi, P. fermentans et Sacch. unisporus sont les seules espèces rencontrées dans la fabrication A et $T r$. capitatum dans la fabrication B. Aucune des 21 souches isolées à partir du lait et du caillé n'assimile le lactose. Les levures isolées à partir du lait et du caillé sont peu résistantes au chlorure de sodium, à l'exception de $T r$. capitatum, espèce capable de se développer en présence de 10 p. 100 de $\mathrm{NaCl}$.

La flore levure du premier affinage

Au cours du premier affinage, $P$. membranaefaciens prédomine à l'intérieur des fromages des deux fabrications (63 p. 100 des isolements). C'est une espèce que n'assimile pas le lactose, mais qui est capable d'utiliser l'acide lactique produit par les streptocoques avant salage. On trouve, en plus faible proportion, des autres levures lactose-négatives. Sur 22 isolements de l'intérieur des fromages pendant le premier affinage, seule une souche de $K$. lactis de la fabrication A est capable d'assimiler (et de fermenter) le lactose. 
La participation des levures à la disparition du lactose de l'intérieur du fromage pendant les 15 premiers jours ne semblent donc pas très importante, compte tenu de la faible proportion de souches lactose-positives. Mais si on considère que la souche de K. lactis a été isolée de la dilution $10^{-6}$, la contribution de cette espèce à l'élimination du lactose n'est pas si négligeable.

En ce qui concerne la production de $\mathrm{CO}_{2}$ par fermentation, le rôle des levures est aussi assez réduit : $K$. lactis est la seule espèce capable de fermenter le lactose isolée à l'intérieur des fromages, et elle n'apparaît que dans une des fabrications.

Nunez et Medina [25] avaient déjà mis en évidence que la contribution des leuconostocs à l'ouverture du caillé était négligeable à cause de leur faible densité de peuplement. Ce changement dans la texture du fromage, qui permettra le développement de $P$. roquefort $i$ pendant le deuxième affinage, est simplement la conséquence de la perte d'eau subie à l'intérieur du fromage pendant le premier affinage (9-10 $\mathrm{g} \mathrm{H}_{2} \mathrm{O} / 100 \mathrm{~g}$ de $100 \mathrm{~g}$ de fromage du $4^{\mathrm{e}}$ au $15^{\mathrm{e}} \mathrm{j}$ ) montrée par Nunez [24].

Nos conclusions différent sensiblement de celles de Devoyod et Sponem [11] pour le fromage de Roquefort et de Millet et al. [23] pour le fromage de Cantal, deux fromages dans lesquels les levures fermentant le lactose (L.F.L.) ont un rôle décisif. Les L.F.L, représentaient dans ces deux fromages respectivement 36 p. 100 et 53 p. 100 des isolements.

Nous croyons que les levures présentes à l'intérieur du fromage de Cabrales pendant le premier affinage contribuent plutôt à la maturation par la consommation de l'acide lactique produit par les streptocoques : toutes les souches isolées l'assimilaient. Le $\mathrm{pH}$ monte du $4^{\mathrm{e}} \mathrm{au} 15^{\mathrm{e}} \mathrm{j}$ parallèlement au nombre des levures : de 4,95 à 6,10 dans le fromage A et de 4,70 à 5,30 dans le fromage $B$.

La flore levure à la surface du fromage pendant le premier affinage est moins dense qu'à l'intérieur, mais plus riche en espèces. Toutes les espèces présentes au centre du fromage se trouvent aussi à la surface. T. candida, espèce qui n'a pas été isolée au centre et qui est capable de se développer avec plus de 10 p. 100 de $\mathrm{NaCl}$, est l'espèce dominante. Les levures de la surface n'assimilaient pas le lactose, à l'exception de 2 souches de $K$. lactis, mais toutes les souches isolées utilisaient l'acide lactique.

\section{La flore levure du deuxième affinage}

A l'intérieur des fromages la population de levures reste élevée jusqu'au $60^{\mathrm{e}} \mathrm{j}$, en dessus de $10^{7} / \mathrm{g}$, puis elle descend lentement. Au centre du fromage, nous trouvons les mêmes espèces que pendant le premier affinage, à l'exception de $K$. lactis. L'espèce $P$. membranaefaciens reste prédominante : 47 p, 100 des isolements. Toutes les souches isolées pendant le deuxième affinage utilisent l'acide 
lactique, n'assimilent pas le lactose et, à part une souche de Sacch. chevalieri, sont peu halotolérantes.

Les levures peuvent participer activement à la maturation du fromage au cours du deuxième affinage par leurs activités protéolytique, lipolytique et de production de composés d'arôme. La majeure partie de l'importante population de levures du premier affinage meurt avant le $90^{\mathrm{e}}$ jour et ses protéases et lipases endocellulaires ont été libérées dans le fromage.

La flore levure de la surface des fromages pendant le deuxième affinage est limitée à deux seules espèces, $D$. hansenii et $T$. candida, très résistantes au chlorure de sodium. Ces résultats coïncident avec ceux de Vergeade et al. [35] sur la flore levure de la croûte du fromage de Saint-Nectaire. Nous avons constaté l'action sélective du $\mathrm{NaCl}$ envers les levures de la surface du fromage de Cabrales, déjà observée par Devoyod et Sponem [11] dans le fromage de Roquefort. Dans le Cabrales, le sel a une action sélective même à niveau de souche : les souches de $D$. hansenii et $T$. candida isolées à la surface du fromage pendant le deuxième affinage sont plus halotolérantes que celles isolées pendant le premier affinage (tab. 3).

Dans quelques autres échantillons, prélevés pendant le deuxième affinage à la surface des fromages de Cabrales de différentes fabrications, on a isolé à partir des dilutions $10^{-4}$ et $10^{-5}$ les espèces Cryptococcus albidus var. albidus, avec quelques souches capables de se développer en présence de 20 p. 100 de $\mathrm{NaCl}$, et Cryp. infirmo-miniatus (Nunez, Medina et Gaya 1979, résultats non publiés).

\section{b) LES MOISISSURES}

\section{Les moisissures du lait et du caillé}

La population de moisissures dans le lait et le caillé est nettement plus basse que celle des levures. G. candidum, la seule espèce isolée, était présente dans le lait et le caillé de la fabrication A, mais pas de la B.

\section{Les moisissures du premier affinage}

Les moisissures prolifèrent au centre du fromage tout au long du premier affinage. $G$. candidum, $P$. roqueforti et $P$. terrestre sont les espèces isolées à l'intérieur. Elles peuvent contribuer à la maturation par la consommation de l'acide lactique, dû à leur nombre : $7,3 \times 10^{7} / \mathrm{g}$ dans la fabrication A et $2,2 \times 10^{6} / \mathrm{g}$ dans la $\mathrm{B}$, le $15^{\mathrm{e}}$ jour.

A la surface du fromage le niveau de moisissures est si réduit que leur contribution à la maturation doit être négligeable. Ce niveau diminue après salage dans le fromage A : l'espèce halosensible $G$. 
candidum, rencontrée dans le lait, le caillé et l'intérieur du fromage n'est pas présente à l'extérieur. Dans le fromage B, par contre, le niveau de moisissures à la surface reste constant pendant le premier affinage.

\section{Les moisissures du deuxième affinage}

$\mathrm{Au}$ cours du deuxième affinage, l'espèce $P$. roqueforti est nettement dominante au centre du fromage, avec 76 p. 100 des isolements. G. candidum est présent à l'intérieur du fromage A jusqu'au $60^{\mathrm{e}} \mathrm{j}$, date à laquelle, d'après Nunez [24], la concentration de $\mathrm{NaCl}$ arrive à 2,3 p. 100 (6,6 p. 100 dans la phase aqueuse). Les espèces P. frequentans et $P$. purpurogenum ont été aussi isolées à l'intérieur du fromage.

Pendant ce stade de la maturation la population de moisissures à la surface du fromage reste également plus basse que celle de l'intérieur. Les espèces $P$. roqueforti et $P$. frequentans représentant la plupart ( 88 p. 100) des isolements. P. terrestre, Penicillium sp. et Paec. varioti ont été isolés occasionnellement à la surface des fromages A et $B$.

Dans d'autres fabrications artisanales de Cabrales $P$. roquefort $i$ était toujours l'espèce prédominante à l'intérieur des fromages pendant le deuxième affinage. La flore des moisissures à la surface de ces fromages était très variable et on a recensé les espèces suivantes : $P$. roquefort $i, P$. terrestre, $P$. cyclopium, $P$. purpurogenum, $P$. lanosum, $P$. camemberti, $P$. commune, $P$. citrinum, $P$. fellutanum, Penicillium spp., G. candidum, Cladosporium herbarum, Aspergillus repens, $A$. sulphureus et Gliocladium roseum. P. roqueforti était généralement présent à la surface en nombre plus élevé que les espèces restantes (Nunez, Medina et Gaya 1979, résultats non publiés).

Les moisissures de la surface du fromage de Cabrales sont donc assez diverses, bien que $P$. roqueforti soit, comme à l'intérieur, l'espèce dominante.

Ces micro-organismes participent activement à la maturation du fromage de Cabrales, principalement à l'intérieur, en éliminant l'acide lactique, en dégradant les protéines et la matière grasse par leurs protéases et lipases exocellulaires et en formant des méthylcétones.

\section{Remerciements}

Nous remercions le $\operatorname{Pr} \mathrm{J}$. Santa Maria pour son aide et ses conseils dans le travail d'identification des levures et $\mathrm{G}$. Jolivet pour sa collaboration dans la rédaction du manuscrit. 


\section{Résumé}

L'évolution quantitative et qualitative de la flore fongique a été étudiée tout au long de la fabrication et de la maturation de deux cuviers de fromage de Cabrales. A partir du lait et du caillé on a isolé les espèces suivantes : Pichia fermentans, Saccharomyces unisporus, Trichosporon capitatum et Geotrichum candidum.

Pendant le premier affinage, du $5^{\mathrm{e}}$ au $15^{\mathrm{e}} \mathrm{j}$, Pichia membranaefaciens prédomine à l'intérieur des fromages et Torulopsis candida à la surface. Ces levures n'assimilent pas le lactose, mais elles contribuent à la maturation en éliminant l'acide lactique. G. candidum et Penicillium roqueforti sont les espèces de moisissures dominantes pendant ce premier affinage.

Au cours du deuxième affinage, du $16^{e}$ au $120^{\mathrm{e}} \mathrm{j}, P$. membranaefaciens et $P$. fermentans représentent la plupart de la flore levure à l'intérieur et Debaryomyces hansenii et $T$. candida, très halotolérants, sont les seules espèces rencontrées à la surface. Parmi les moisissures, $P$. roqueforti est l'espèce qui prédomine nettement au centre du fromage, tandis que $P$. roqueforti et $P$. frequentans sont les principales espèces de la surface.

\section{S u m mary}

THE YEASTS AND MOULDS OF SPANISH MOULD-RIPENED

CABRALES CHEESE

Changes in number and composition of fungic flora have been investigated throughout the cheese-making and ripening processes of two batches of Cabrales cheese. The following species were isolated from milk and curd: Pichia fermentans, Saccharomyces unisporus, Trichosporon capitatum and Geotrichum candidum.

During the first ripening (5-15 days), Pichia membranaefaciens was the yeast species which predominated in the interior of cheeses and Torulopsis candida on the cheese surface. They did not assimilate lactose, but utilized lactic acid, rising the $\mathrm{pH}$ and contribuing so to the ripening process. G. candidum and Penicillium roqueforti were the main mould species during this period.

Throughout cave ripening (16-120 days), P. membranaefaciens and $P$. fermentans were the majority yeast flora in the interior of cheeses and halotolerant species Debaryomyces hansenii and $T$. candida the only ones found on the cheese surface. $P$. roqueforti was the main mould species in the interior of cheeses, while $P$. roqueforti and $P$. frequentans predominated on the cheese surface.

Reçu pour publication en septembre 1980. 


\section{Bibliographie}

[1] Accolas (J. P.), Melcion (D.) and Vassal (L.) (1978). - Study on the surface microflora of Gruyère and Beaufort cheeses. $X X$ Int. Dairy Congr., vol. E, 762.

[2] Ainsworth (G. C.), Sparrow (F. K.) and Sussman (A. S.) (1973). - The Fungi Vols. 4 A, B. Academic Press, London.

[3] American public health association (1978). - Standard Methods for the Examination of Dairy Products, 14 ed. A.P.H.A., New York.

[4] Augusto (G.), Luigi (M. P.) e Adriano (C.) (1968). - Microflora of Robbiola cheese. Latte, 42, 871. (Dairy Sci. Abstr., 32, 3453).

[5] Bodini (T.), Guicciardi (A.) e Craveri (R.) (1969). - Microbiological and chemical study of some soft cheeses, and the identification of the yeasts and fungi. Latte, 43, 711. (Dairy Sci. Abstr., 32, 3452).

[6] Carini (S.), Kaderavek (G.), Gregori (A. de) e InVernizzi (F.) (1971). Robbiola cheese and its chemical and microbiological characteristics. Latte, 45, 615. (Dairy Sci. Abstr., 34, 4225).

[7] Dale (G.) (1972). - Moisissures et levures de la flore du fromage de SaintNectaire. Rev. Lait. Fr., 296, 199.

[8] Dale (G.) et Gutllot (J.) (1971). - Contribution à l'étude de la microflore du fromage de Saint-Nectaire et de la physiologie du Geotrichum candidum Link. Compt. rend. des séances de la Société de Biologie, 165, 309.

[9] Deiana (P.), Fatichenti (F.) e Farris (G. A.) (1977). - Indagini microbiologiche sul latte e sul formaggio di capra in Sardegna. Nota I : I lieviti. Ind. del latte, 13, 49.

[10] Delespaul (G.), Gueguen (M.) et Lenoir (J.) (1973). - La flore fongique superficielle des fromages de Saint-Nectaire et de Tome de Savoie. I. Son évolution au cours de l'affinage. Rev. Lait. Fr., 313, 715.

[11] Devoyod (J. J.) et Sponem (D.) (1970), - La flore microbienne du fromage de Roquefort. VI. Les levures. Le Lait, 50, 524.

[12] Ducastelle (A.) et Lenoir (J.) (1965). - Contribution à l'étude de la flore microbienne du fromage de type Saint-Paulin. II. Ses espèces dominantes Le Lait, 45, 509.

[13] Engel (G.) and Teuber (M.) (1978). - Simple aid for the identification of Penicillium roqueforti Thom. European J. Appl. Microbiol., 6, 107.

[14] Fatichenti (F.), Farris (G. A.) e Deiana (P.) (1977). - L'évoluzione della microflora blastomicetica nel «Fiore Sardo». Ind. del Latte, 13, 11.

[15] Galzin (M.), Galzy (P.) et Bret (G.) (1970). - Etude de la flore de levure dans le fromage de Roquefort. Le Lait, 50, 1.

[16] Georgantas (St.) (1979). - The blastomycetic flora during the ripening of Telemes cheese. Milchwissenschaft, 34, 24.

[17] Harrigan (W. F.) and Mc CANCE (M.) (1976). - Laboratory Methods in Food and Dairy Microbiology. Academic Press, London.

[18] HaRtley (G. B.) and Jezeski (J. J.) (1954), - The microflora of blue cheese slime. J. Dairy Sci., 37, 436.

[19] Kanauchi (T.), Yoshioka (Y.) and Hamamoto (M.) (1962), - Microbial studies on blue veined cheese. I. Microflora of blue veined cheese in ripening period. Jap. J. Zootech. Sci., 32, 104.

[20] LenorR (J.) (1962). - La flore microbienne du Camembert et son évolution au cours de la maturation. C.R. Acad. Agric., 48, 392. 
[21] Lodder (J.) (1970). - The Yeasts. North Holland Publishing Company, Amsterdam.

[22] Mc Clary (D. O.), Nulty (W. L.) and Miller (G. R.) (1959). - Effect of potassium versus sodium in the sporulation of Saccharomyces. J. Bacteriol., 78, 362 .

[23] Millet (L.), Melcion (D.) et Devoyod (J. J.) (1974), - La flore microbienne du fromage de Cantal fabriqué à partir de lait cru. III. Rôle des levures dans la maturation de la «Tome ». Le Lait, 54, 616.

[24] Northolt (M. D.) and Soentoro (P. S. S.) (1979). - Fungal species on Dutch cheese. Neth. Milk Dairy J., 33, 205.

[25] Nunez (M.) (1978). - Microflora of Cabrales cheese: changes during maturation. J. Dairy Res., 45, 501.

[26] Nunez (M.) et Medina (M.) (1979). - La flore lactique du fromage bleu de Cabrales. Le Lait, 59, 497.

[27] Nunez (M.) et Medina (M.) (1980). - Les microcoques et les staphylocoques dans le fromage bleu de Cabrales. Le Lait, 60, 171.

[28] Ottogalli (G.), Bianchi (B.), Roldinini (G.), Sarachi (S.), Resmini (P.), Galli (A.), Salvadori (P.) e Volonterio (G.) (1971). Chemical and microbiological study of ripening of Gorgonzola cheese. Latte, 45, 776. (Dairy Sci. Abstr., 34, 4226).

[29] Piergiovanni (L.) e Volonterio (G.) (1978). - Influenza della microflora superficiale su alcune caratteristiche del formaggio Provolone. Ind. del Latte, 3, 41.

[30] Raper (K. B.) and Thom (C.) (1949). - Manual of the Penicillia. Baillière, Thindall and Cose, London.

[31] Rosini (G.) (1976). - I lieviti in formaggi «Pecorino» ottenuti con cagli tradizionali e microbici. Ann. Fac. Agr. Perugia, 31, 435.

[32] SAMSON (R. A.), EcKardT (C.) and ORTH (R.) (1977). - The taxonomy of Penicillium species from fermented cheeses. Antonie van Leeuwenhoek, $43,341$.

[33] Sala (F. J.) y Burgos (J.) (1972). - Maduracion del queso "Cabrales». Microflora: III. Levaduras. Anales Bromatol., 24, 83.

[34] Sala (F. J.), Burgos (J.) y Ordonez (J. A.) (1971). - Maduracion del queso «Cabrales». Microflora: II. Mohos. Anales Fac. Vet. Leon, 17, 115.

[35] Schmidt (J. L.) et Lenorr (J.) (1978). - Contribution à l'étude de la flore levure du fromage de Camembert. Son évolution au cours de la maturation. Le Lait, 58, 355.

[36] Vergeade (J.), Guiraud (J.), Larpent (J. P.) et Galzy (P.) (1976). - Etude de la flore de levure du Saint-Nectaire. Le Lait, 56, 275.

[37] VIZZARDI (M.) (1975). - Microbiological study of the ripening of Taleggio cheese in mountain huts in Valsassina. Riv. del Latte, 31, 18. (Dairy Sci. Abstr., 38, 8125).

[38] Wickerham (L. J.) (1946). - A critical evaluation of the nitrogen assimilation test commonly used in the classification of yeasts. J. Bacteriol., 52, 293.

[39] Wickerham (L. J.) (1951). - Taxonomy of yeasts. Tech. Bull. U.S. Dept. Agric., No. 1029.

[40] Wickerham (L. J.) and Burton (K A.) (1948). - Carbon assimilation tests for the classification of yeasts. J. Bacteriot., 56, 363 . 